\title{
Prospective Study of Proportions and Causes of Cancellation of Surgical Operations at Jimma University Teaching Hospital, Ethiopia
}

Haile $\mathrm{M}^{1 *}$, Nega Desalegn ${ }^{2}$

${ }^{1}$ Lecturer and Senior Anesthetist, Department of Anesthesia, Jimma University, Ethiopia.

${ }^{2}$ College of Public Health and Medical Sciences, Department of Anesthesia, Jimma University, Ethiopia.

\begin{abstract}
Background: Cancellation of scheduled surgery is a major quality of health care problem affecting the individual patients, family and the actual health care organization.

Objective: The aim of this study was to assess the incidence, causes and magnitude of cancellation of elective surgical operations and to find the appropriate solutions for better patient management and effective utilization of resources.

Methods: A longitudinal study design was conducted at Jimma University Teaching Hospital from February 1, 2014 to June30, 2014. All consecutive scheduled cases $(n=1438)$ to undergo elective surgical procedures were included in the study.

Result: A total of 1438 patients were scheduled for elective surgical operations. Of these, 331(23.0\%) were cancelled. about $45.6 \%$ male and $54.4 \%$ female ware not operated on the intended day of schedule respectively. General surgery had the highest rate of cancellations $198(23 \%)$ followed by orthopedic surgery $78(20 \%)$. In appropriate scheduling and unavailability of sterile drapes and lab sheets were the main causes of cancelation.

Conclusion and Recommendation: Inappropriate scheduling and unavailability of sterile clothes were the main causes of Cancellation of elective surgical operations in our hospital. Concerned bodies should bring a sustainable change and improvement to prevent unnecessary cancellations and enhance cost effectiveness through communications, careful planning and efficient utilization of the available hospital resources.
\end{abstract}

Keywords: Cancellation; Theater; Surgical; Operations; Ethiopia.

\section{*Corresponding Author:}

Merga Haile,

Lecturer and Senior Anesthetist, Department of Anesthesia, Jimma University, Ethiopia.

E-Mail:merga_haile@yahoo.com

Received: February 07, 2015

Accepted: March 10, 2015

Published: March 11, 2015

Citation: Haile M, Nega Desalegn (2015) Prospective Study of Proportions and Causes of Cancellation of Surgical Operations at Jimma University Teaching Hospital, Ethiopia. Int J Anesth Res. 3(2), 87-90. doi: http://dx.doi.org/10.19070/2332-2780-1500022

Copyright: Haile $\mathbf{M}^{\odot}$ 2015. This is an open-access article distributed under the terms of the Creative Commons Attribution License, which permits unrestricted use, distribution and reproduction in any medium, provided the original author and source are credited.

\section{Introduction}

The operation theatre has been reported to be the heart of a hospital requiring considerable human resources and expenditure from the hospital budget [1].

Most hospitals in developed countries invest considerable re- sources in maintaining operating suites and having surgeons and theatre staff available on an agreed schedule [2]. However, in developing countries where resources are limited, cancellation of elective surgical operations due to various preventable reasons is a common phenomenon in most hospitals $[2,3]$. It is well recognized that cancellation of patients from elective theatre operating lists increases cost, decreases efficiency, duplicates workload and wastes operating room time $[1,4]$. Cancellation of elective surgical procedures also causes significantly emotional trauma to the patients as well as their families and the community in general, and its impact on hospital resources is great due to prolonged hospitalization and high cost of health care $[1,5,6]$. Elective surgery cancellations always lead to insufficient utilization of manpower and hospital resources and can also lead to an increase in patients' treatment expenses due to prolonged hospital stay and in many cases, repetitions of pre-operative preparations and management $[6,7]$.

Different definitions of cancellation exist in the international literature .Some authors define 'cancellation' as only those procedures that were cancelled on the day on which surgery was scheduled [8], whereas others also include those that were cancelled on the previous day $[9,10]$. The Modernization Agency Theatre Programme (National Health Service (NHS), UK) appears to define cancellations as those that occur after the patient has been 
notified of operation date [11].

Some other studies group reasons for cancellations into relatively broad categories, while others simply list causes without grouping them $[12,13]$. In yet other studies, the underlying decision to cancel is explored (e.g. whether anesthetist, the surgeon, patient or hospital initiated the cancellation) [11, 14]. The Australian Council of Healthcare Standards [15] divides cancellations in day surgery into 'failure to arrive' and 'cancellation of the procedure after arrival. Repeat cancellations have implications for patient satisfaction, staff morale, hospital-patient relationship and training [4, 6, 7]. This study was designed to assess the magnitude and causes for cancellation as a first step in identifying measures to reduce them.

\section{Methodology}

\section{Study Design and Setting}

A longitudinal study design was conducted from February 1, 2014 to June 30, 2014 in Jimma University Teaching Hospital, which is found in Jimma city, Southwest Ethiopia $350 \mathrm{~km}$ away from the capital city of the country. It has a total of 450 beds, three major operation theaters and 558 health professionals, where a multidisciplinary team of diverse professionals provide a range of health care services for approximately 9,000 inpatients and 80,000 outpatients each year.

The three Major operation theaters provide sub-specialty service for General surgical procedures, Obstetrics and gynecologic surgery, ophthalmic procedures, maxillofacial surgeries and orthopedic procedures.

Overall the operation theaters consists of five operation tables, from these two tables used by General surgery, gynecology and maxillofacial surgeries on different days; Monday and Wednesday gynecological surgeries, Tuesday and Thursday by general surgery and Friday maxillofacial surgeries, Usually no room is reserved for emergency cases ,hence emergency surgeries were given priority on each day. Orthopedic procedures have one separate operation table which functions the whole week. Obstetric and ophthalmic procedures have isolated operation units.

In our set up all patients are seen by the surgeon/senior resident a day before surgery either in the ward or at the OPD clinic and usually fully investigated, prepared according to the diagnosis. The operating list is prepared by the resident surgeons, verified by the head, and sent to the theatre. The head surgeon confirms book- ings and supervises scheduling activities. Operative cancellations were defined as those cases that were booked in the operative list and did not have the planned surgery on the intended date.

\section{Study Subjects}

The study subjects were all undergoing operation on elective bases. Emergency cases and a minor surgical procedure performed in outpatient department were not included.

All patients cancelled in the Pre-Operative Holding area were candidate for enrollment. A cancellation form, which contains the patient's demographic data (age \& gender), the diagnosis, type of surgical procedure, causes of cancellations of the procedure was filled for each case.

Information regarding the cancellation of surgeries was gathered from various sources including; the operating room daily surgical schedule, preoperative anesthetic assessment form, physicians, the anesthetist performed preoperative evaluation, the anesthetist responsible for conducting the anesthesia techniques and head theater nurses. Data collected were collated and analyzed using descriptive statistics.

\section{Ethical Clearance}

Ethical approval was obtained from the Jimma university ethical review committee before the commencement of the study.

\section{Result}

A total of 1438 patients scheduled to undergo elective surgical procedures during the study period. Of these, 1107 (77.0\%) patients were operated on the intended date of schedule while the remaining 331 cases were cancelled on the day of surgery for various reasons, giving an overall cancellation rate of $23.0 \%$. Of 678 $(47.14 \%)$ male and $760(52.86 \%)$ female patients scheduled for the operation, about $45.6 \%$ male and $54.4 \%$ female ware not operated on the intended day of schedule respectively. Of the total number of patents whose operation was cancelled general surgery takes the majority 198 (23\%) followed by orthopedic surgery 391 $(20 \%)$.

Common reasons for elective surgical patient cancelation is inappropriate scheduling $(33.5 \%)$ followed by lack of sterile drape $(23.5 \%)$ and in appropriate patient preparation $(11.8 \%)$.

\section{Discussion}

Table 1. Distribution of scheduled surgical procedures and cancellations per specialties.

\begin{tabular}{|c|c|c|c|}
\hline \multirow{2}{*}{ Surgical service } & $\begin{array}{c}\text { Scheduled surgical } \\
\text { procedures }\end{array}$ & \multicolumn{2}{c|}{ Cancelled Surgical Procedures } \\
\cline { 2 - 4 } & Operations (n) & Cancelled Operations(n) & Cancellation rate \\
\hline General Surgery & 863 & 198 & 23 \\
\hline Orthopedics & 392 & 78 & 20 \\
\hline Gynecology & 130 & 38 & 29.2 \\
\hline ophthalmology & 40 & 12 & 30 \\
\hline Maxillofacial & 14 & 5 & 35.7 \\
\hline
\end{tabular}


Figure 1. Percentage distribution of causes of elective surgical patient cancelations.

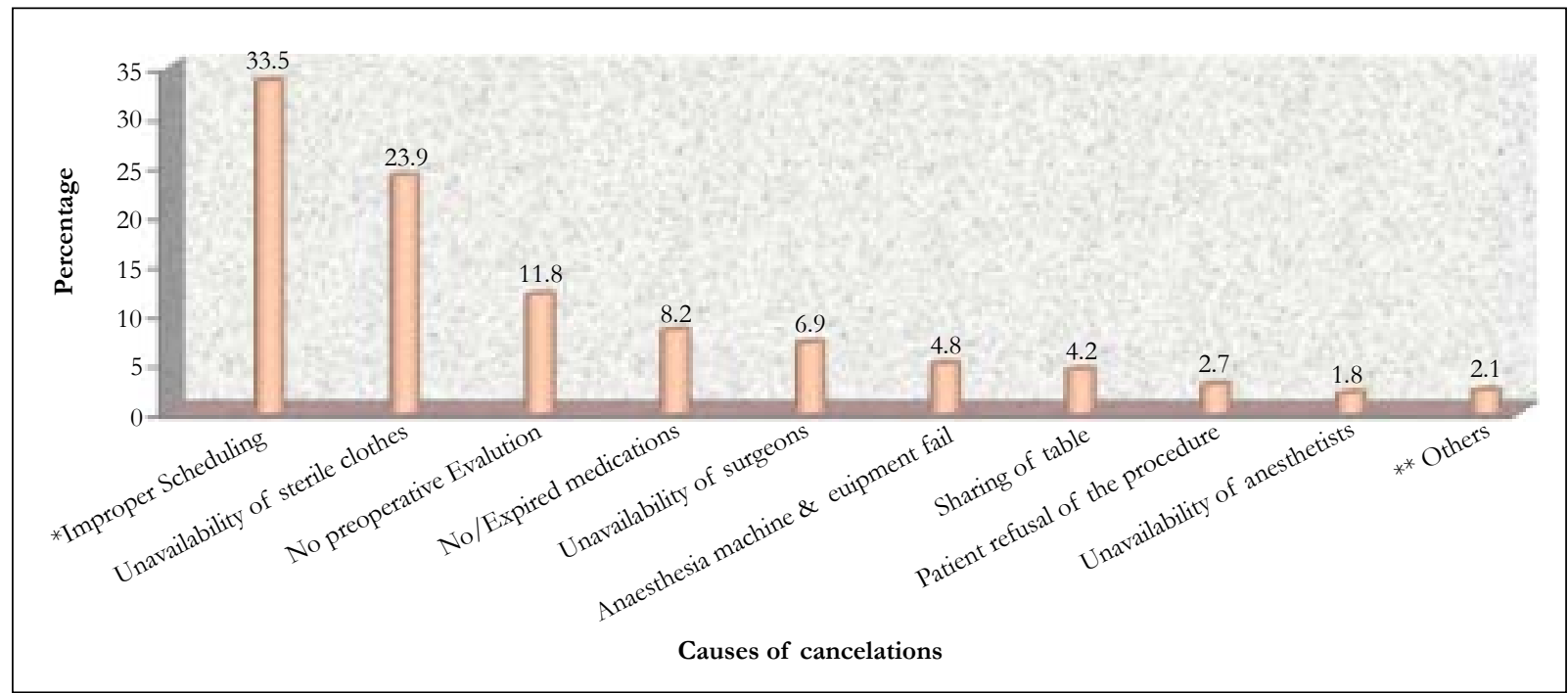

*improper scheduling: (long operation lists/ Time constraints, uncontrolled medical illness, inadequate theatre staff) **Others: ( Financial constraints, no blood available etc)

Increasing patient satisfaction through efficient practice is an appropriate objective of a health care system. A high cancellation rate for elective surgical procedures makes it difficult to accomplish this [16]. Cancellation reduces operating room efficiency and increases costs $[17,18]$. It also requires major expenditure of time and cost by patients and their families [1, 5, 6, 22]. Cancellation of an elective procedure carries a major cost implication to the hospitals because of wasted hospital resources and major impact on patients because of loss of time from work as well as the impact of the wasted effort on the patient's family [22].

The acceptable cancellation rate for elective surgical procedures is controversial [7]. The reported incidences for elective surgery cancellation vary widely among institutions from $10-40 \%[4,7,8$, 19-21].

In our study, the rate of cancellation of elective surgical procedures was $23 \%$ which is higher than what was reported by Zafar A [1] and Lacqua MJ [19] and similar with the studies done at Tanzania teaching hospital (21\%) and Ayub teaching hospital (25\%). This difference could be improper scheduling (33.5\%), (long operation lists/ Time constraints, uncontrolled medical illness, inadequate theatre staff); lack of sterile drapes for surgery (23.9\%) and in appropriate patient preparation [figure 1].

Cancellation among different surgical subspecialties general surgery accounts about $23 \%$ followed by orthopedic and gynecologic procedures $(20 \%, 29.2 \%)$ respectively. These figures are almost comparable with study done at University Teaching Hospital in the Lake Zone, Tanzania showed cancellation of General surgery (31.5\%), Orthopedic (25.5\%), Gynecologic (24.3\%), some of the difference in this figures could be due to sample size of our study, staffing and communications and other administration associated.

Cancellations of operation due to inadequate preparation of important medical conditions are avoidable $[5-7,22]$ the medical problems could have been identified in time and the number of cancellations on medical grounds could have been avoided by establishing a formal protocol with the surgeons. All the patients are assessed a day before operation by the anesthetist in our hos- pital and the few available anesthesiologist were not involved in preoperative assessment.

A good administration and good team communication can reduce cancelation rates, this is because many of reasons for elective surgical cancelations in our set up were avoidable and more of admistrative issue and communication related. Lengthy operation schedule lists prepared by junior surgeons/ interns, who were not familiar with the procedure, was also a reason for cancellation of operations.

We observed influence of surgeon experience in this study and found inexperienced surgeons add significantly to the operation time this is because of our hospital is teaching hospital and we observed residents of different level get involved in the surgical procedures as part of their education. Consultants were quicker and their presence reduced the likelihood of complications, thus reduced operative time. Moreover it was observed that if a consultant surgeon the list is likely to proceed with fewer cancelations.

\section{Conclusion and Recommendation}

The incidence of cancellation of elective surgical procedures on the scheduled day of surgery is high in our hospital. Most of the causes of the cancellations are potentially preventable. In order to enhance cost-effectiveness and efficiency, efforts should be made to prevent unnecessary cancellations through careful planning and effective communications.

A long-term planning should be aimed at increasing operation theatre spaces equipped with enough facilities and manpower.

\section{Acknowledgements}

Author thanks Jimma university operation unit staffs and study participants.

\section{References}

[1]. Zafar A, Mufti TS, Griffin S, Ahmed S (2007) Cancelled elective general operations in Ayub Teaching hospital. J. Ayub Med Coll Abbollabad 19 (3): 
64-66

[2]. Garg R, Bhalotra AR, Bhadoria P, Gupta N, Anand R (2009) Reasons for cancellation of cases on the day of surgery: A Prospective study IndianJournal of Anesthesia 53 (1): 35-39

[3]. Schofield WN, George L.R, Michael P, Ying Y.L, Doungkamol S, et al. (2005) Cancellation of operation on the day of intended surgery at a major Australian referral hospital Med J Australia 182 (12): 612- 615

[4]. Robb WB, O'Sullivan MJ, Brannigan AE, Bouchier-Hayes DJ (2004) Are elective surgical operations cancelled due to increasing medical admissions? Irish J Med Sci 173(3):129.

[5]. Miller GG (2004)Waiting for an operation: parent's perspectives. Can J Surg 47(3):167-9.

[6]. Kolawole I.K, Bolaji B.O (2002) Reasons for cancellation of elective surgery in Ilorin. Nig .J Surg. Res 4 (1-2): 28-33

[7]. Ojo E.O, Ihezue C.H (2008) An Audit of Day Case Cancellations In A Nigerian Tertiary Hospital Based Day Case Unit East and Central African Journal of Surgery 13 (2); 150-153

[8]. Rai MR, Pandit JJ (2003) Day of surgery cancellations after nurse led preassessment in an elective surgical centre: the first 2 years. Anesthesia 58: 692-9.

[9]. Henderson BA, Naveiras M, Butler N, Hertzmark E, Ferrufino-Ponce Z (2006) Incidence and causes of ocular surgery cancellations in an ambulatory surgical center. Journal of Cataract and Refractive Surgery 32: 95-102

[10]. Jimenez A, Artigas C, Elia M, Casamayor C, Gracia JA, et al. (2006) Cancellations in ambulatory day surgery: Ten years observational study. Journal of Ambulatory Surgery 12: 119-23.

[11]. (2002) National Health Service, Modernization Agency. Theatre Programme. Step Guide to Improving Operating Theatre Performance. London: NHS.

[12]. Griffin XL, Griffin DR, Berry AR, Hunter DC (2006) Cancellation of elective surgery - any improvement after ten years? Annals of The Royal College of Surgeons of England 88:28-30.
[13]. Report by the Comptroller and Auditor General for Northern Ireland. The Use of Operating Theatres in Northern Ireland Health and Personal Social Services. Northern Ireland Audit Office, 9 April 2003.http://www.niauditoffice.gov.uk/(accessed 6 October 2008).

[14]. Van Klei WA, Moons KGM, Rutten CLG (2002) The effect of outpatient preoperative evaluation of hospital inpatients on cancellation of surgery and length of hospital stay. Anesthesia and Analgesia 94: 644-9.

[15]. Australian Day Surgery Council. Day Surgery/Endoscopy Indicators: Clinical Indicators - A Users' Manual, version 3.http://www.surgeons.org (accessed 3 September 2008).

[16]. Ivarsson B, Larsson S, Sjo “berg T (2004) Postponed or cancelled heart operations from the patient's perspective. Journal of Nursing Management 12 : 28-36

[17]. McIntosh C, Dexter F, Epstein RH (2006) The impact of service specific staffing, case scheduling, turnovers, and first-case starts on anesthesia group and operating room productivity: a tutorial using data from an Australian hospital. Anesthesia and Analgesia 103: 1499-516.

[18]. Pandit JJ, Westbury S, Pandit M (2007). The concept of surgical operating list 'efficiency': a formula to describe the term. Anaesthesia; 62: 895-903.

[19]. Lacqua MJ, Evans JT (1994) Cancelled elective surgery: an evaluation. American Surgeon 60:809-11

[20]. Dakum N. K, Ramyil V. M, Misauno M. A, Ojo E.O, Ogwuche E. I, et al. (2006) A. Reasons for cancellations of urologic day care surgery.Nigerian of Surgical Research $8(1-2)$ : 30-33.

[21]. El-Bushra A.D, Mohamed I.M, Awadalla M.A, Mohamed Y.B, Salah E.M (2008) Cancelled elective surgical operations at El Obeid Hospital, Western Sudan. Sudan Med. J 44 (1, 2\&3):56-61.

[22]. Nasr A. Reichardt K, Fitzgerald K (2004) Arumugusamy M. Keeling P. Walsh T. N. Impact of emergency admissions on elective surgical workload Irish J. Med Sci 173 (3): 133-135 